\title{
Type-2 fuzzy logic controller optimized by wavelet networks for mobile robot navigation
}

Fatma Affane, Kadda Zemalache Meguenni, Abdelhafid Omari

LDEE, Université des Sciences et de la Technologie d'Oran-Mohamed Boudiaf, Algeria

\begin{tabular}{|c|c|}
\hline Article Info & ABSTRACT \\
\hline Article history: & In this work, we will use a new control strategy based on the integration of a \\
\hline Received Mar 17, 2019 & $\begin{array}{l}\text { type- } 2 \text { fuzzy reasoning optimized by wavelet networks as part of a navigation } \\
\text { system of a mobile robot. The proposed approach is able to facilitate the }\end{array}$ \\
\hline Revised Jul 7, 2019 & navigation task in an autonomous manner, in order to determine which \\
\hline Accepted Sep 2, 2019 & $\begin{array}{l}\text { commands must be sent at each moment to the mobile robot. This operation } \\
\text { must take into account convergence towards a goal with the shortest possible }\end{array}$ \\
\hline Keywords: & $\begin{array}{l}\text { path in the minimum delay between the starting position and the target } \\
\text { position. Once the goal is reached, the robot stops. }\end{array}$ \\
\hline
\end{tabular}

Mobile robot

Navigation

Optimization

Type- 2 fuzzy logic controller

Wavelets

Copyright $\odot 2020$ Institute of Advanced Engineering and Science. All rights reserved.

Corresponding Author:

Fatma Affane,

Department d'Automatique

Université des Sciences et de la Technologies d'Oran (USTO-MB), Algerie.

Email: fatma.affane@univ-usto.dz

\section{INTRODUCTION}

The theory of fuzzy logic has been established by L.zadeh [1]. This logic allows the representation and processing of inaccurate or approximate knowledge. It is expressed by a set of linguistic rules called fuzzy rules, which are used to control complex systems or scarcely modeled [2-4]. The number of applications based on fuzzy logic theory in the field of mobile robotics has increased significantly in recent years [5-12].

Since fuzzy systems are built from the knowledge provided by the human expert, they are tainted with uncertainties. These uncertainties are injected into the membership functions of the fuzzy antecedent and consequent sets that will be uncertain. These fuzzy systems, called fuzzy type-1 systems, are incapable of modeling these uncertainties because they use specific membership functions, which have a two-dimensional representation. Therefore, fuzzy type- 2 systems, whose membership functions themselves are unclear, are the extension of type-1 fuzzy systems. In recent years, several works have been developed based on type-2 fuzzy systems. They are used inimage processing [13-15], the control of electrical machines [16-19], and the control of mobile robots [20-23]. But the disadvantage of fuzzy logic is the empirical choice of parameters, which can make the control of the system long and delicate in certain situations.

This problem prompted researchers to propose methods for the automatic optimization of certain parameters of the fuzzy controller, we can quote the work of [24] who designed an evolutionary algorithm to optimize the type 2 fuzzy controller and used it for tracking control of autonomous mobile robots trajectory, there is also the work of [25] who proposed a new method namely the uncontrolled genetic sorting algorithm for optimizing a proportional-integral-derivative type-2fuzzy logic controller for the follow-up control of trajectory of a Delta parallel robot. In the work [26], the authors controlled the cooperation of the robots and the tasks of reaching the target when navigating for several mobile robots using a type-2 fuzzy logic controller optimized by the PSO method. Our goal is to optimize type-2 fuzzy logic controller formobile 
robot navigation. To perfect our goal we have opted for the use of wavelet networks that combine the capacity of neural networks in learning and those of wavelets in the decomposition of signals.

Our work is broken into two parts the first is to use two type-2 fuzzy systems, one to generate the control in angular velocity and the other for the linear speed of the robot to achieve the convergence of the mobile robot towards a goal. The second part concerns the optimization of controllers applied using wavelet networks.

\section{RESEARCH METHOD}

\subsection{Proposed Navigation Strategy}

The strategy adopted here for the navigation of the robot determines a movement order allowing the robot to move so that it can achieve its goal, from the current position of the robot and the definition of the position of the target point. This action is performed in an environment where no obstacle hinders the progress of the robot. Fuzzy logic reasoning of type- 2 is used. Parameters of the robot and its environment shown in Figure 1.

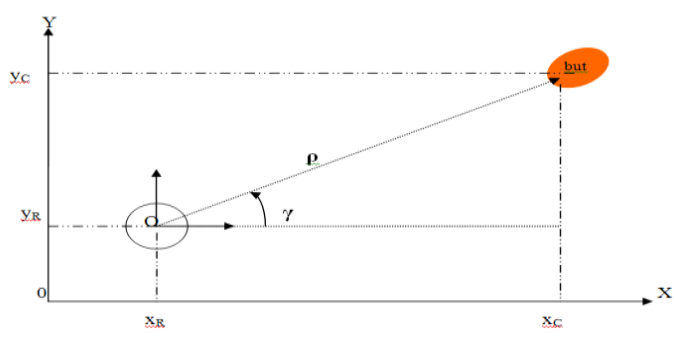

Figure 1. Parameters of the robot and its environment

\subsection{Synthesis of the first Type-2 Fuzzy Logic Controller T2FLC1}

This controller receives as input the orientation of the target $(\boldsymbol{\gamma})$ and its variation $(\Delta \gamma)$, and returns the output command $\Delta \theta$, which corresponds to the change in the orientation of the robot.

\subsubsection{Membership Functions}

The speech universes of the two inputs are decomposed respectively into fivetype- 2 fuzzy subsets for $\gamma$, andthree for $\Delta \gamma$ which are represented by: LB (Left Big), LS (Left Small ), Z (Zero), RS (Right Small), RS (Right Big),N (Negative), Z (Zero), and P (Positive) as shown in Figure 2 and 3.

The labels used for the fifteen fuzzy subsets relating to the change of orientation are:

NVB (Négative Very Big), NB (Négative Big), NM (Négative Medium), NMS (Négative Medium Small), NS (Négative Small ), NVS (Négative Very Small), NVVS (Négative Very Very Small), ZE (Zéro), PVVS (Positive VeryVerySmall), PVS(PositiveVerySmall), PS(Positive Small), PMS (Positive Medium Small), PM (Positive Medium), PB (Positive Big), and PVB (Positive Very Big).
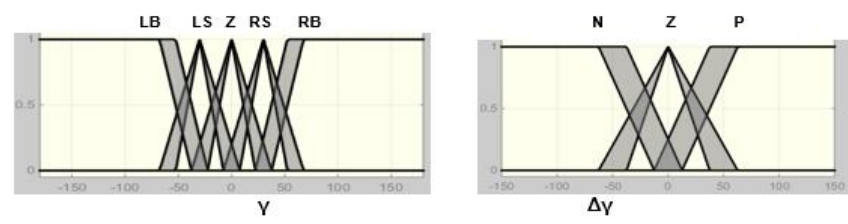

Figure 2. Membership functions of input variables

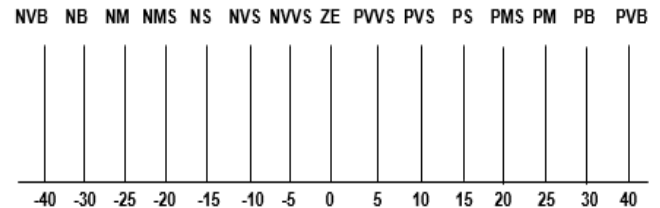

Figure 3. Membership functions of the output variable $\Delta \theta$

\subsubsection{Fuzzy Rules Base}

The following base of the rules summarizes, in Table 1 is the variation of the orientation as a function of the angle as well as its variation. It symbolizes the following relationship: the variation of direction that the robot must adopt is all the greater as the point of arrival is located largely on the sides of the robot:

Type-2 fuzzy logic controller optimized by wavelet networks for mobile robot navigation (Fatma Affane) 


\begin{tabular}{lccccc}
\multicolumn{5}{c}{ Table 1. Fuzzy Rules Base } \\
\hline & LB & LS & Z & RS & RB \\
\hline N & PVB & PMS & PVVS & NVS & NM \\
Z & PB & PS & ZE & NS & NB \\
P & PM & PVS & NVVS & NMS & NVB \\
\hline
\end{tabular}

\subsection{Synthesis of the Second Type-2 Fuzzy Logic Controller T2FLC2}

This controller receives two inputs, the first $\rho$ represents the distance between the robot and the target, and the second input represents the linear speed of the instantaneous robot $v$. The output returned by this controller represents the speed variation $\Delta \mathrm{v}$.

\subsubsection{Membership Functions}

The speech universes of the inputs are decomposed into two, and five type-2 fuzzy subsets of, which can be respectively represented by: N (Near), F (Far) for $\rho$ and VS(Very Small), S (Smal), M (Medium), $\mathrm{B}$ (Big), and VB (Very Big) for v as shown in Figure 4 and 5.

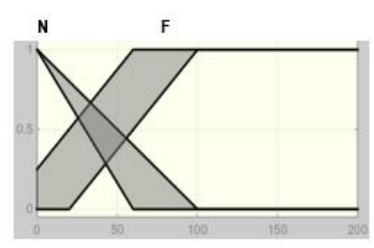

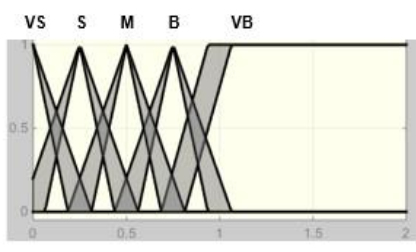

$\mathbf{v}$

Figure 4. Membership functions of input variables

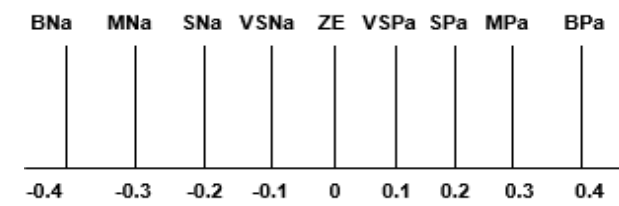

Figure 5. Membership functions of the output variable $\Delta \mathrm{v}$

The labels used for the nine (9) relating to the subsets of the output $\Delta v$ are: BNa (Big Negative acceleration), MNa (Medium Negative acceleration), SNa (Small Negative acceleration), VSNa (Very Small Negative acceleration), ZE (Zero), VSPa (Very Small Positive acceleration), SPa (Small Positive acceleration), MPa(Medium Positive acceleration), and BPa (Big Positive acceleration).

\subsubsection{Fuzzy Rules Base}

The following rule base summarizes the variation of the speed (acceleration) as in Table 2 is a function of the current speed $\mathrm{v}$ and the distance $\rho$. It symbolizes the following relationship: the robot's acceleration is all the greater as the distance between the robot and the target is high and the robot's current speed is average.

Table 2. Fuzzy Rules Base

\begin{tabular}{cccccc}
\hline & VS & S & M & B & VB \\
\hline N & ZE & VSN_a & SN_a & MN_a & BN_a \\
F & BP_a & BP_a & MP_a & SP_a & VSP_a \\
\hline
\end{tabular}

\subsection{Optimization of Synthesized Type-2 Fuzzy Logic Controllers}

In our work, we use the wavelet network which presents interesting performances in identification and predictive control [27], and we show how it can be used to optimize the parameters of a type-2 fuzzy logic controller at the premise part and the consequent part by adjusting the parameters of a wavelet function.

\subsubsection{Principle of the Method}

This method presents a complete structural analogy with a fuzzy inference system of the SUGENO type(SIF). The latter (SIF) can be schematized in the form of a multilayer network whose connections are weighted at its output, and the square nodes are adaptive. The membership functions used are of triangular shape, which leads to the development of an algorithm that is suitable for this type of function. The wavelet network architecture used is shown in Figure 6. 


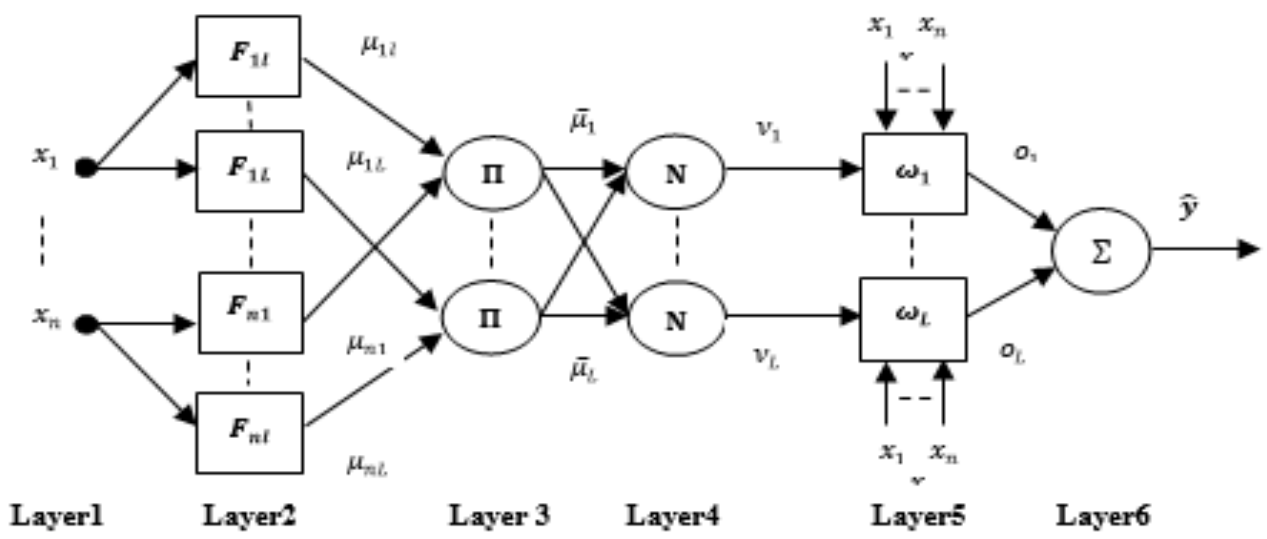

Figure 6. Wavelet network architecture used [5]

This network therefore comprises six layers:

Layer 1: Receiving the inputs and transmitting to the next layer.

Layer 2: a proper triangular membership function for each node $\mathrm{F}_{\mathrm{i} \ell}$

$$
\mu_{i \ell}=1-\frac{2 \cdot\left|x_{\ell}-a_{i \ell}\right|}{b_{i \ell}}
$$

Where $a_{i \ell}$ and $b_{i \ell}$ are the centers and basic widths of the triangular function ofthe $l^{\text {th }}$ term ofthei ${ }^{\text {th }}$ ith input variable respectively.

Layer 3:Multiplication of all input signals:

$$
\overline{\mu_{\ell}}=\prod_{i=1}^{n} \mu_{i \ell}
$$

Layer 4: several fixed nodes labeled by $\mathrm{N}$, whose the ith node calculates this ratio:

$$
v_{\ell}=\frac{\overline{\mu_{\ell}}}{\sum_{\ell=1}^{L} \overline{\mu_{\ell}}}
$$

Layer 5: these nodes are of adaptive type and they calculate the function $\boldsymbol{\omega}_{\boldsymbol{\ell}}$ expressed by:

$$
\omega_{\ell}=\left(\sum_{i=1}^{n} \emptyset_{i \ell}\right) w_{\ell}=\left(\sum_{i=1}^{n}-\vartheta_{i \ell} \exp \left(\frac{-\left(\vartheta_{i \ell}\right)^{2}}{2}\right)\right) w_{\ell}
$$

where $\emptyset_{i l}$ is the mother wavelet function (first derivative of the Gaussian function )

$$
\vartheta_{i \ell}=\frac{x_{i}-m_{i \ell}}{d_{i \ell}}
$$

with $m_{i \ell}$ and $d_{i \ell}$ are the translation and expansion coefficients of this wavelet and $w_{\ell}$ is the weighting parameter of this node. The output of the layer is expressed by the relation

$$
o_{\ell}=\omega_{\ell} v_{\ell}
$$

Layer 6: contains a single neuron that calculates the sum of all the signals coming from the fifth layer according to the following formula:

$$
\hat{y}=\sum_{\ell=1}^{L} o_{\ell}=\sum_{\ell=1}^{L} \omega_{\ell} v_{\ell}
$$

\subsubsection{Optimization Algorithm}

The gradient descent method is used to adjust the parameters $a_{i \ell}, b_{i \ell}, m_{i \ell}, d_{i \ell}$ and $w_{\ell}$ whose parameter adaptation equations are obtained by:

$$
a_{i \ell}(k+1)=a_{i \ell}(k)+\eta(y(k)-\hat{y}(k)) \frac{\partial \hat{y}(k)}{\partial a_{i \ell}(k)}
$$




$$
\begin{aligned}
& b_{i \ell}(k+1)=b_{i \ell}(k)+\eta(y(k)-\hat{y}(k)) \frac{\partial \hat{y}(k)}{\partial b_{i \ell}(k)} \\
& m_{i \ell}(k+1)=m_{i \ell}(k)+\eta(y(k)-\hat{y}(k)) \frac{\partial \hat{y}(k)}{\partial m_{i \ell}} \\
& d_{i \ell}(k+1)=d_{i \ell}(k)+\eta(y(k)-\hat{y}(k)) \frac{\partial \hat{y}(k)}{\partial d_{i \ell}(k)} \\
& w_{\ell}(k+1)=w_{\ell}(k)+\eta(y(k)-\hat{y}(k)) \frac{\partial \hat{y}(k)}{\partial w_{\ell}(k)}
\end{aligned}
$$

where

$$
\begin{aligned}
& \frac{\partial \hat{y}(k)}{\partial a_{i \ell}}=\frac{\partial \hat{y}(k)}{\partial v_{\ell}} \frac{\partial v_{\ell}}{\partial \mu_{i \ell}} \frac{\partial \mu_{i \ell}}{\partial a_{i \ell}}=\left(O_{\ell}(k)-\hat{y}(k) v_{\ell} \operatorname{signe}\left(x_{i}-a_{i \ell}\right) \cdot \frac{2}{b_{i \ell} \mu_{i \ell}}\right. \\
& \frac{\partial \hat{y}(k)}{\partial b_{i \ell}}=\frac{\partial \hat{y}(k)}{\partial v_{\ell}} \frac{\partial v_{\ell}}{\partial \mu_{i \ell}} \frac{\partial \mu_{i \ell}}{\partial b_{i \ell}}=\left(O_{\ell}(k)-\hat{y}(k)\right) v_{\ell} \frac{1-\mu_{i \ell}}{b_{i \ell} \mu_{i \ell}} \\
& \frac{\partial \hat{y}(k)}{\partial m_{i \ell}}=\frac{\partial \hat{y}(k)}{\partial \omega_{\ell}} \frac{\partial \omega_{\ell}}{\partial \phi_{i \ell}} \frac{\partial \phi_{i \ell}}{\partial v_{i \ell}} \frac{\partial v_{i \ell}}{\partial m_{i \ell}}=v_{\ell} w_{\ell} \phi_{i \ell} \frac{\left(\vartheta_{i \ell}^{2}-1\right)}{\vartheta_{i \ell} d_{i \ell}} \\
& \frac{\partial \hat{y}(k)}{\partial d_{i \ell}}=\frac{\partial \hat{y}(k)}{\partial \omega_{\ell}} \frac{\partial \omega_{\ell}}{\partial \phi_{i \ell}} \frac{\partial \phi_{i \ell}}{\partial \vartheta_{i \ell}} \frac{\partial v_{i \ell}}{\partial d_{i \ell}}=v_{\ell} w_{\ell} \phi_{i \ell} \frac{\left(\vartheta_{i \ell}^{2}-1\right)}{d_{i \ell}} \\
& \frac{\partial \hat{y}(k)}{\partial w_{\ell}}=\frac{\partial \hat{y}(k)}{\partial \omega_{\ell}} \frac{\partial \omega_{\ell}}{\partial w_{l}}=v_{\ell}\left(\sum_{i=1}^{n}-\vartheta_{i \ell} \exp \left(\frac{-\left(\vartheta_{i \ell}\right)^{2}}{2}\right)\right)
\end{aligned}
$$

\section{RESULTS AND ANALYSIS}

Figure 7 is presented simulating the evolution of the robot in the environment so that it can reach the target by controlling the change of its orientation and the variation of its speed taking into account several different situations for the robot and the target using the two type-2 fuzzy logic controllers.
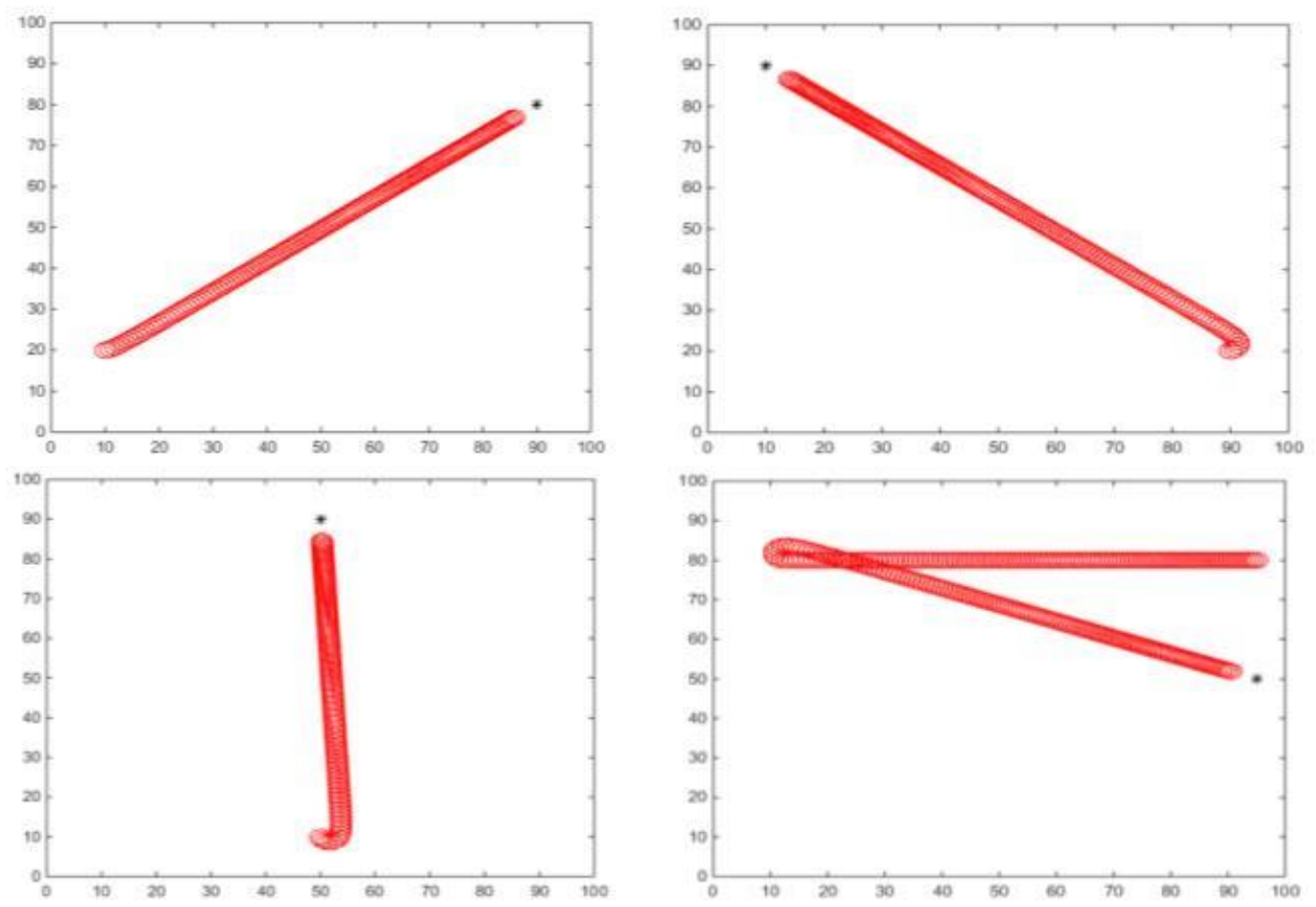

Figure 7. Simulation examples 
Consider now the optimization of type-2 fuzzy logic controllers applied to generate the control of the change of orientation $\Delta \theta$ and of the speed variation $\Delta \mathrm{v}$, gives the following Table 3 .

Table 3. Tables Obtained

\begin{tabular}{cccccc}
\hline \multicolumn{7}{c}{ a) T2FLC1 } \\
\hline & LB & LS & Z & RS & \multicolumn{2}{c}{ RB } \\
\hline $\mathrm{N}$ & 49.55 & 3.41 & 8.41 & -6.5 & $28{ }^{-} 6$ \\
$\mathrm{Z}$ & 33.26 & 18.5 & 3.5 & -11.48 & -26.54 \\
$\mathrm{P}$ & 21.73 & 13.5 & -1.49 & -16.48 & -32.17 \\
\hline
\end{tabular}

b) T2FLC2

\begin{tabular}{cccccc}
\hline & $\mathrm{VS}$ & $\mathrm{S}$ & $\mathrm{M}$ & $\mathrm{B}$ & $\mathrm{VB}$ \\
\hline $\mathrm{N}$ & -0.08 & -0.13 & -0.28 & -0.39 & -0.33 \\
$\mathrm{~F}$ & 0.46 & 0.29 & 0.19 & 0.09 & -0.14 \\
\hline
\end{tabular}

We can now give a linguistic interpretation to this table. For that, we assign the concept PVB (PositiveVery Big) to numerical values greater than 40, the concept PB (Positive Big) with numerical values between 27 and 37, the PM (PositiveMedium) concept with numerical values between 22 and 27, the concept PMS (Positive MediumSmall) with numerical values between 17 and 22, the concept PS (Positive Small) with numerical values between 12 and 17, the concept PVS (Positive Very Small) with numerical values between 7 and 12, the concept PVVS (Positive Very VerySmall) with numerical values between 3 and 7 , and the concept ZE (Zero) with values close to 0 , and likewise in the negative sense for the first controller.

And with regard to the second controller, we assign the conceptBPa (Big Positive acceleration)to numerical values greater than 0.38 , the concept $\mathrm{MPa}$ (Medium Positive acceleration)with numerical values between 0.25 and 0.35, the concept of SPa (Small Positive acceleration) with numerical values between 0.15 and 0.25 , the concept hasVSPa (Very Small Positive acceleration)with numeric values between 0.07 and 0.15 , and the conceptZE (Zero)with values close to 0 , and likewise in the negative sense. We obtain the following tables:

Table 4. Linguistic Interpretation of the Tables Obtained a) T2FLC1

\begin{tabular}{clcccc}
\hline & LB & LS & Z & RS & RB \\
\hline N & PVB & PM & PVS & NVVS & N \\
Z & PB & PMS & PVVS & NVS & NM \\
P & PMS & PS & ZE & NS & NB \\
\hline
\end{tabular}

\begin{tabular}{cccccc}
\multicolumn{5}{c}{ b) T2FLC2 } \\
\hline & $\mathrm{VS}$ & $\mathrm{S}$ & $\mathrm{M}$ & $\mathrm{B}$ & $\mathrm{VB}$ \\
\hline $\mathrm{N}$ & $\mathrm{ZE}$ & $\mathrm{VSNa}$ & $\mathrm{MNa}$ & $\mathrm{BNa}$ & $\mathrm{MNa}$ \\
$\mathrm{F}$ & $\mathrm{BPa}$ & $\mathrm{MPa}$ & $\mathrm{SPa}$ & $\mathrm{ZE}$ & $\mathrm{VSNa}$ \\
\hline
\end{tabular}

The tables obtained are different from those proposed by the human expert, which modifies the behavior of the rules. If we compare them to tables provided by the expertise, we see that they are not homogeneous. The behavior of the rules is not close to that of fuzzy controllers proposed for the same task by a human expert. The shaded boxes in Table 4 are the difference between the two tables (For example NB replaces $\mathrm{NM}$ in the first table and $\mathrm{ZE}$ replaces $\mathrm{SPa}$ in the second table). We note that these tables are not close to those given in Tables 1 and 2, which means that the strategy extracted by the fuzzy logic controllers optimized is removed from that proposed by the human expert. The optimized membership functions of the input variables for both type-2 fuzzy controllers are shown in Figure 8 and 9.
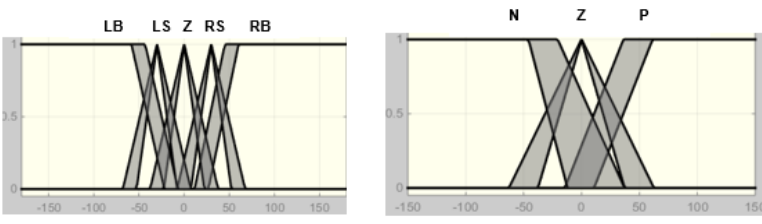

Figure 8. The optimized membership functions of the T2FLC1
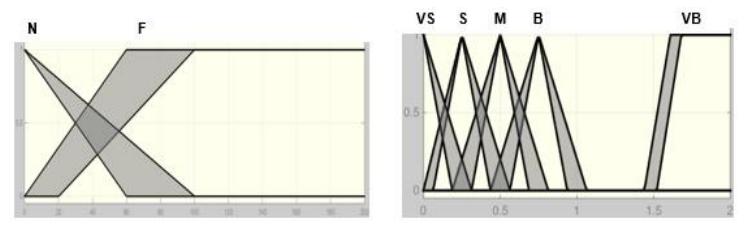

Figure 9. The optimized membership functions of the T2FLC2

To illustrate the performance of these controllers optimized by wavelet networks, we performed the same simulation tests made with the type-2 fuzzy controllers previously synthesized. These results confirm those obtained previously and show that the optimization method followed makes it possible to obtain readable rules tables that are easy to interpret. To illustrate the performance of these optimized controllers, the same examples are used.

Type-2 fuzzy logic controller optimized by wavelet networks for mobile robot navigation (Fatma Affane) 
From the results of the simulation conducted, it can be seen that the results obtained with the type-2 fuzzy controllers optimized by the wavelet networks are better compared to those obtained with the nonoptimized controllers in the direction of the change of orientation of the mobile robot which is done in a manner faster. In addition, the wavelet network method has the advantage of optimizing the membership functions and the fuzzy rules simultaneously. Results of the simulation conducted as shown in Figure 10.
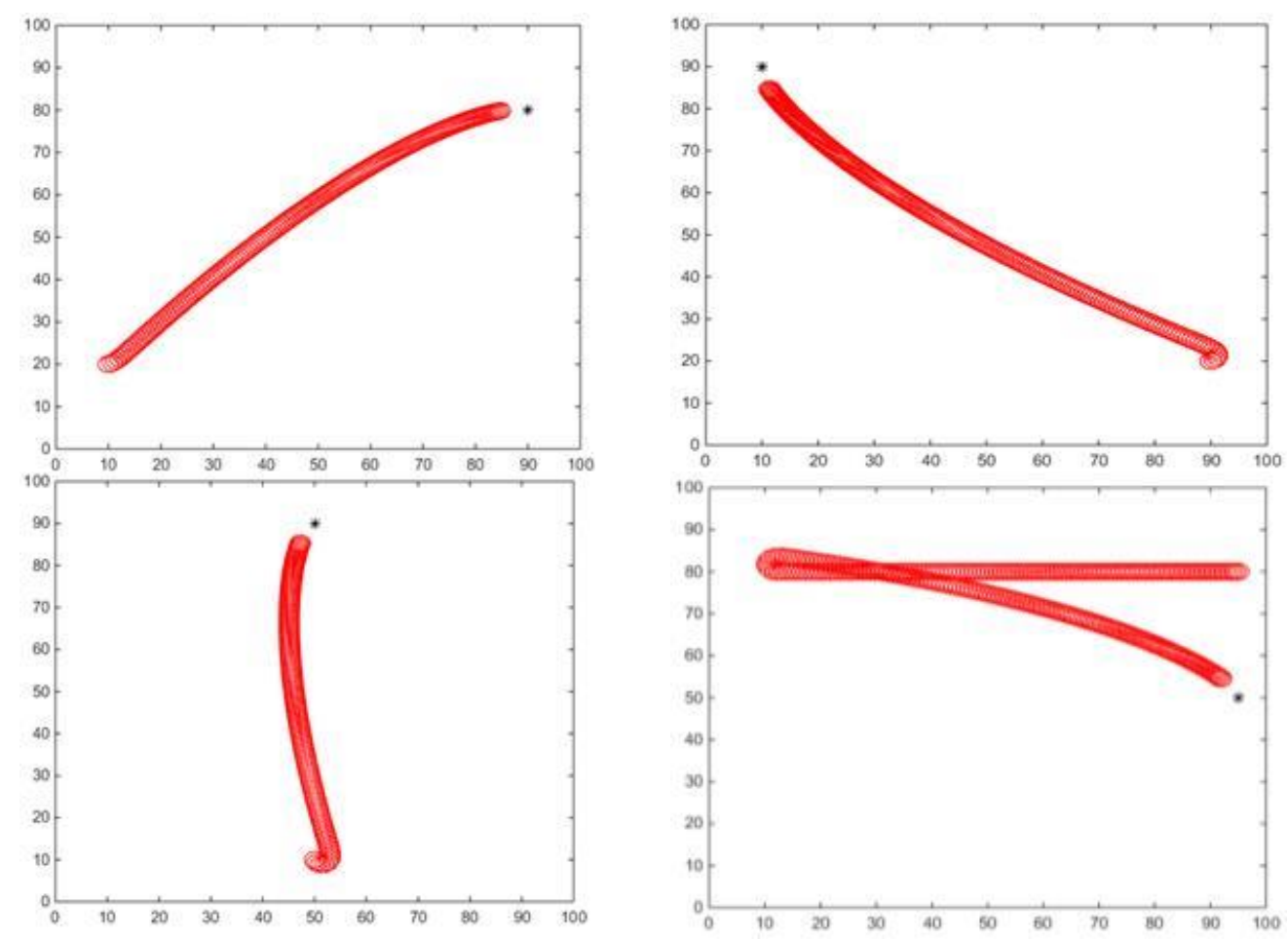

Figure 10. Results of the simulation conducted

\section{CONCLUSION}

In this paper, we presented a new optimization technique based on the integration of a type-2 fuzzy controller with wavelet networks for mobile robot navigation. This approach has the advantage of optimizing membership functions and fuzzy rules simultaneously.

We navigated a mobile robot using two optimized type-2 fuzzy logic controllers, one for generating the change of orientation command and the other for the speed variation. These controllers must take into account convergence towards a goal with the shortest possible path in the minimum delay between the starting position and the target position. We gave a linguistic interpretation to thesetables.

From the results obtained with type-2fuzzy logic controllers optimized by wavelet networks, we can say that these results are better compared to those obtained with the expertise. We also noticed that this new optimization technique has resulted in readable, easy-to-interpret rule tables.

\section{REFERENCES}

[1] A Zadeh LA, " The Concept of a Linguistic Variable and its Application to Approximate Reasoning-I," ELSEVIER Information Science, vol.8-3, pp. 199-249, 1975.

[2] Nabil Farah, M. H. N. Talib, Z. Ibrahim, J. M. Lazi, Maaspaliza Azri, "Self-tuning Fuzzy Logic Controller Based on Takagi-Sugeno Applied to Induction Motor Drives," International Journal of Power Electronics and Drive System, vol.9-4, pp. 1967-1975, 2018.

[3] Abdulrahman Moffaq Alawad, Farah Diyana Abdul Rahman, Othman O. Khalifa, Norun Abdul Malek, "Fuzzy Logic based Edge Detection Method for Image Processing," International Journal of Electrical and Computer Engineering , vol.8-3, pp. 1863-1869, 2018.

[4] Mohd Faisal Farhan, Nor Sakinah Abdul Shukor, Mohd Ashraf Ahmad, Mohd Helmi Suid, Mohd Riduwan Ghazali, Mohd Falfazli Mat Jusof, "Implicit force control approach for safe physical robot-to-human object handover," Indonesian Journal of Electrical Engineering and Computer Science, vol.14-2 , pp. 903-911, 2019. 
[5] Ahmad TaherAzar, Hossam Hassan Ammar, HazemMlik, "Fuzzy Logic Controller with Color Vision System Trackingfor Mobile Manipulator Robot,"The International Conference on Advanced Machine Learning Technologies and Applications AMLTA. Advances in Intelligent Systems and Computing, Springer , vol.723, pp. 138-146, 2018.

[6] Asita Kumar Ratha,Dayal R,Parhib, Harish Chandra Dasc, Manoj Kumar Munib,PriyadarshiBiplabKuma, "Analysis and use of fuzzy intelligent technique for navigation ofhumanoid robot in obstacle prone zone Defence Technology," vol.14-6, pp. 677-682, 2018.

[7] MaïssaBoujelben, ChokriRekik, Nabil Derbel, "A hybrid fuzzy-sliding mode controller for a mobile robot,".Int. J. Modelling, Identification and Control, vol. 25-3 , pp. 155-164, 2016.

[8] Rahib, H Abiyev, Irfan, S Günsel, Nurullah Akkaya, Ersin Aytac, Ahmet Çağman, Sanan Abizada, " Fuzzy control of omnidirectional robot," Elsevier 9th International Conference on Theory and Application of Soft Computing, Computing with Words and Perception ICSCCW , vol.120 , pp. 608-616, 2017.

[9] Thoa T, Mac, CosminCopot, Robin De Keyser, Trung D. Tran, Thich Vu, "MIMO Fuzzy Control for Autonomous Mobile Robot,"Journal of Automation and Control Engineering , vol.4-1 , pp. 65-70, 2016.

[10] ZatiHanani Ismail1, Syibrah Naim2, Ahmad Faisal Ayob3 and AzamCheAmat, "Mobile Robot Controller Based on Fuzzy Logic System in Uneven Terrain," 9th International Conference on Theory and Application of Soft Computing, Computing withWords and Perception, ICSCCW , Menemui Matematik, vol.39-2 , pp. 70-82, 2017.

[11] Agung Nugroho Jati, Randy Erfa Saputra, M. Ghozy Nurcahyadi, Nasy’an Taufiq Al Ghifari, "A Multi-robot System Coordination Design and Analysis on Wall Follower Robot Group," International Journal of Electrical and Computer Engineering , vol.8-6, pp. 5078-5106, 2018.

[12] Ade Silvia Handayani1, Siti Nurmaini, Irsyadi Yani, Nyayu Latifah Husni, "Analysis on swarm robot coordination using fuzzy logic," Indonesian Journal of Electrical Engineering and Computer Science , vol.13-1 , pp. 48-57, 2019.

[13] DibyaJyoti, Bora, R. S. Thakur, "An Efficient Technique for Medical Image Enhancement Based on Interval Type2 Fuzzy Set Logic," Progress in Computing, Analytics and Networking. Advances in Intelligent Systems and Computing, Springer, Singapore, vol.710, pp. 667-678, 2018.

[14] Vikas Singh, Raghav Dev, Narendra K. Dhar, Pooja Agrawal, Nishchal K. Verma, "Adaptive Type-2 Fuzzy Approach for Filtering Salt and Pepper Noise in Grayscale Images, "IEEE Transactions on Fuzzy Systems, vol.26-5, pp. 3170-3176, 2018.

[15] Mehmet Karakose, Hasan Yetis, SemihaMakinist, "Image Processing-Based Center Calculation Method for General and Interval Type-2 Fuzzy Systems.International Journal of Fuzzy Systems,", vol.20-5, pp. 1699-1712, 2018.

[16] BelabbasBelkacem M, Tadjine, AllaouiTayeb, MouloudDenai, " Higher Performance of the Type 2 Fuzzy Logic Controller for Direct Power Control of Wind Generator Based on a Doubly Fed Induction Generator in Dynamic Regime,"International Conference in Artificial Intelligence in Renewable Energetic Systems ICAIRES, vol.35, pp. 215-223, 2018.

[17] HosseinHassani, JafarZarei. " Interval Type-2 fuzzy logic controller design for the speed control of DC motors," Journal Systems Science \& Control Engineering, vol.3-1 , pp. 266-273, 2015.

[18] MoseGalluzzo, Bartolomeo Cosenza . "Control of a non-isothermal continuous stirred tank reactor by a feedbackfeedforward structure using type-2 fuzzy logic controllers," Information Sciences, vol.181-17 , pp. 3535-3550, 2011.

[19] Agung B. Muljono1, I. M. Ginarsa, I. M. A. Nrartha, A. Dharma. " Coordination of Adaptive Neuro Fuzzy Inference System (ANFIS) and Type-2 Fuzzy Logic System-Power System Stabilizer (T2FLS-PSS) to Improve a Large-scale Power System Stability Type-2 Fuzzy Approach for Filtering Salt and Pepper Noise in Grayscale Images," International Journal of Electrical and Computer Engineering , vol.8-1 , pp. 76-86, 2018.

[20] DorraAyedi,Maissa Boujelben,ChokriRekik. "Hybrid Type-2 Fuzzy-Sliding Mode Controller for Navigation ofMobile Robot in an Environment Containing a Dynamic Target,"Journal of Robotic , pp. 1-10, 2018.

[21] Krishna Kant Pandey, Anish Pandey, AnimeshChhotrayandDayal R. Parhi. "Navigation of Mobile Robot Using Type-2FLC," Proceedings of the International Conference on Signal, Networks, Computing, and Systems, Springer, New Delhi,vol.396, pp. 137-145, 2016.

[22] Mouloud IDER, Boubekeur MENDIL. "Type-2 fuzzy logic control for a mobile robot tracking a moving target," Mediterranean Journal of Modeling and SimulationMJMS, vol.3 , pp. 057-065, 2015.

[23] NesrineBaklouti, Robert John, Adel M. Alimi. " Interval Type-2 Fuzzy Logic Control of Mobile Robots," Journal of Intelligent Learning Systems and Applications , vol.4: , pp. 291-302, 2012.

[24] Ricardo Martinez and Antonio Rodriguez, Oscar Castillo, Patricia Melin, Luis T Aguilar. " Optimization of Type-2 Fuzzy Logic Controllers for Mobile Robots Using Evolutionary Methods,"IEEE International Conference on Systems, Man, and Cybernetics San Antonio,2009, pp. 4764-4769.

[25] Xingguo Lu, Ming Liu. "Optimal Design and Tuning of PID-type Interval Type-2 Fuzzy Logic Controllers for Delta Parallel Robots, "International Journal of Advanced Robotic Systems.; , vol. 13(3): , pp. 1-12, 2016.

[26] Allawi ZT, Abdalla TYA. "A PSO-Optimized Type-2 Fuzzy Logic Controller for Navigation of Multiple Mobile Robots." IEEE 19th International Conference on Methods and Models in Automation and Robotics MMAR,2014, pp. 33-39.

[27] Chi-Huang Lu. "Wavelet Fuzzy Neural Networks for Identification and Predictive Control of Dynamic Systems, " IEEE Transactions On Industrial Electronics. 2011, vol.58-7, pp. 3046 - 3058 


\section{BIOGRAPHIES OF AUTHORS}

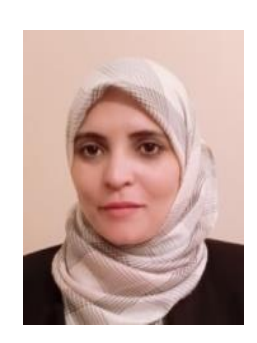

Fatima Affane Received her B.Eng, degree and the M.S degree in Automatic Engineering from University of Sciences and Technology of Oran, Algeria. Currently, she is preparing her $\mathrm{PhD}$ in Automatic from the same University.

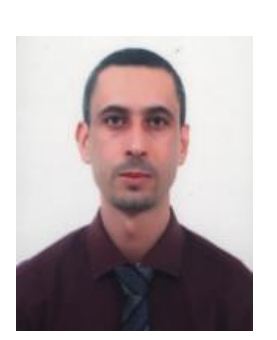

Kadda Zemalache Meguenni Received his B.S degree and the M.S degree in Electrical Engineering from University of Sciences and Technology of Oran, Algeria, in 1998, and 2001 respectively and his $\mathrm{PhD}$ in Electrical Engineering from University of Evry Val d'Essonne, France, in 2006. He is currently working as an Associate Professor in the Faculty of Electrical Engineering at the University of Sciences and Technology in the city of Oran in Algeria. His research and teaching interests include: Intelligent control, Mechatronics systems and Robotics control.

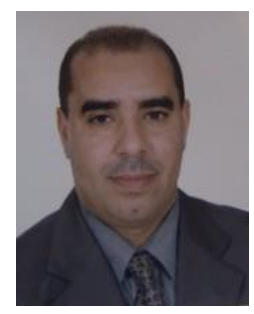

Abdelhafid Omari obtained his MSc from the University of Sciences and Technology of Oran in Algeria in 1992 and the $\mathrm{PhD}$ in Electrical Engineering from the University of Electrocommunications Tokyo, Japan in 2001. He worked in academia and industry. His industrial experience was mainly in the field of mechatronics and robotics. He is currently with the department of automatic control in the Faculty of Electrical Engineering at the University of Sciences and Technology of Oran in Algeria. His research and teaching interests include: linear and nonlinear control methods, robust control design and intelligent mechatronic systems. 